\title{
ZNF703 acts as an oncogene that promotes progression in gastric cancer
}

\author{
GONGLI YANG ${ }^{1,2 *}$, FENG MA ${ }^{1 *}$, MUXIAO ZHONG $^{1}$, LIN FANG $^{1}$, YAO PENG $^{1}$, XIAOMING XIN $^{1}$, \\ JIETAO ZHONG $^{1}$, FANGFANG YUAN ${ }^{1}$, HONGXIANG GU ${ }^{1}$, WEI ZHU ${ }^{1}$ and YALI ZHANG ${ }^{1}$ \\ ${ }^{1}$ Guangdong Provincial Key Laboratory of Gastroenterology, Department of Gastroenterology, Nanfang Hospital, \\ Southern Medical University, Guangzhou, Guangdong 510515; ${ }^{2}$ Department of Gastroenterology, \\ Taihe Hospital, Hubei Medical University, Shiyan, Hubei 442000, P.R. China
}

Received November 18, 2013; Accepted January 8, 2014

DOI: $10.3892 /$ or.2014.2997

\begin{abstract}
ZNF703, a member of the NET/Nlz family of zinc finger transcription factors, contributes to aspects of developmental growth and patterning across evolutionarily diverse species. ZNF703 has been identified as a novel oncogene in human breast cancer. In the present study, we investigated the expression of ZNF703 in gastric carcinoma and attempted to determine, using cell line models, its biological actions. Using immunohistochemistry, we analyzed the ZNF703 protein expression in 120 clinicopathologically characterized gastric cancer cases. Using RNA interference, we investigated the effects of ZNF703 depletion on tumor proliferation and metastasis in vitro. We found that ZNF703 was overexpressed in invasive gastric carcinoma tissues, and its expression levels were closely correlated with the depth of invasion, node metastasis and venous invasion. RNA interference-mediated silencing of the ZNF703 gene in SGC7901 cells inhibited cell proliferation and migration significantly. The results showed that ZNF703 acts as a gastric cancer oncogene and should be considered a therapeutic target for metastatic gastric cancer.
\end{abstract}

\section{Introduction}

Gastric carcinoma, the fourth most common malignancy and the second most frequent cause of cancer-related mortality, is the result of accumulated genomic damage, affecting cellular functions essential for cancer development (1). Despite recent advances in combination chemotherapies (2), the outcome of

Correspondence to: Professor Wei Zhu or Professor Yali Zhang, Guangdong Provincial Key Laboratory of Gastroenterology, Department of Gastroenterology, Nanfang Hospital, Southern Medical University, 1838 Guangzhou North Avenue, Baiyun, Guangzhou, Guangdong 510515, P.R. China

E-mail: chnz_w@126.com

E-mail: sygznfyy@163.com

${ }^{*}$ Contributed equally

Key words: gastric carcinoma, ZNF703, oncogene, metastasis unresectable gastric cancer remains poor. The deep invasive carcinomas, as in gastric carcinoma, have higher rates of lymph duct and venous invasion and lymph node metastasis (3). However, the mechanism of invasion and metastasis of gastric carcinoma is not fully understood.

Members of the recently identified NET (for nocA, Nlz, elB and TLP-1) protein family have emerged as crucial for developmental growth and patterning across evolutionarily diverse species (4). In humans, two NET family members, NLZ1/ZNF703 [also known as FLJ14299, AK024361, Zeppo1 (Zpo1) and henceforth referred to as NocA-like zinc finger 1 (NLZ1) and NLZ2/ZNF503 (NLZ2)] have been identified (5-8). ZNF703 is located on chromosomes 8 (8p11.23). Several studies have associated gene amplification of the 8p11-12 chromosomal region with human breast cancer (9). Holland et al used genomic approaches to show that 8p12 was amplified in $13 \%$ of luminal B breast cancers. They reasoned that ZNF703 was likely to be the most functionally important gene in the $8 \mathrm{p} 12$ amplicon, as it was the only gene found to be amplified in two unique luminal B breast tumors (10). Two recent independent studies identified ZNF703 as the associated novel oncogene $(10,11)$. The single-nucleotide polymorphism (SNP) rs7816345 near ZNF703 has strong links to breast cancer (12). Furthermore, overexpression of mouse ortholog of ZNF703 (Nlz1/Zeppo1) has been associated with breast cancer progression and metastasis in mice (13).

To date, however, the role played by ZNF703 in the development and progression of gastrointestinal malignant tumor is unclear. In the present study, the expression of ZNF703 was examined in primary human gastric adenocarcinomas. Studies were also carried out on gastric carcinoma cell lines aimed at determining the biological functions of ZNF703.

\section{Materials and methods}

Cell lines. Gastric cancer cell lines (AGS, SGC7901, MGC823 and BGC802) were obtained from the China Center for Type Culture Collection (CCTCC, Wuhan, China). All the cells were maintained in RPMI-1640 medium (HyClone, Logan, UT, USA) supplemented with $10 \% \mathrm{FBS}$ at $37^{\circ} \mathrm{C}$ in $5 \% \mathrm{CO}_{2}$. The cells were passaged with Trypsin-EDTA (0.05\% trypsin and $0.53 \mathrm{mM}$ tetrasodium EDTA). Protein and RNA samples 
were extracted from subconfluent cells in the exponential phase of growth.

Patients and tumor samples. Formalin-fixed tumor tissues from the 120 patients including 25 paired normal mucosa were used for immunohistochemical analysis. From the 120 patients, fresh frozen tumor tissues and paired normal mucosa $(6 \mathrm{~cm}$ from the tumor) were randomly selected from 12 patients for protein analysis (western blotting) and mRNA analysis [reverse transcription-polymerase chain reaction (RT-PCR)]. All the patients who underwent curative resection for primary gastric adenocarcinomas were operated on at Nanfang Hospital during the period 2009-2012. Each tumor was assigned a histological type and a depth grading of infiltration according to the Lauren classification (14), Goseki et al $(15,16)$ and the World Health Organization classification (17). The examination was performed on routine slides to identify lymphatic and venous invasion. The hematoxylin and eosin staining method was used in all cases. Each histological parameter was determined when invasion and/or metastasis was identified with certainty. Diagnosis was established by two independent pathologists. All the tissue specimens for the present study were obtained following patient informed consent. The collection of these tissue samples was carried out with the approval of the Ethics Committee of Southern Medical University.

Immunohistochemistry analysis. Formalin-fixed and paraffin embedded tissues were cut into $4 \mu \mathrm{m}$ sections, deparaffinized in xylene and rehydrated in phosphate-buffered saline. Deparaffinized sections were pre-incubated with normal bovine serum and then incubated overnight at $4^{\circ} \mathrm{C}$ with an optimal dilution (1:500) of a primary polyclonal rabbit antibody against human ZNF703 (GTX107721; GeneTex, Irvine, CA, USA). The slides were sequentially incubated with a biotinylated goat anti-rabbit immunoglobulin $\mathrm{G}$ antibody, and the reaction products were visualized using diaminobenzidine (DAB; Dako, Carpinteria, CA, USA) with methyl green as a counterstain. For negative control, the primary antibodies were omitted, but otherwise the methodology was the same. ZNF703 expressions were classified into three categories depending on the percentage of cells stained:,$- 0-10 \%$ positive cells;,$+ 10-50 \%$ positive tumor cells; and,$++>50 \%$ positive tumor cells.

Western blot analysis. Western blot analysis was performed on human gastric adenocarcinoma tissues and gastric adenocarcinoma cell lines. The tissues and cells were then suspended in RIPA buffer (50 mM Tris, $150 \mathrm{mM} \mathrm{NaCl}, 1 \%$ NP-40, $1 \%$ sodium deoxycholate and $0.05 \%$ SDS, $\mathrm{pH} 7.4$ ). The supernatant was collected, and the protein concentration was quantified using a protein assay reagent (Bio-Rad Laboratories, Hercules, CA, USA). After boiling, the proteins (25 $\mu \mathrm{g})$ were separated by polyacrylamide gel electrophoresis (PAGE) under denaturing, and transferred to a Hybond ECL nitrocellulose membrane (Amersham Biosciences, Arlington Heights, IL, USA). The membranes were blocked with 5\% low-fat dried milk in TBS containing 0.1\% Tween-20 (TBS-T), and then incubated for $1 \mathrm{~h}$ at room temperature with 1:1,000 dilutions of the antihuman ZNF703 antibody (GTX107721). We processed anti-human $\beta$-actin antibody (N-21; Santa Cruz Biotechnology, Inc., Santa Cruz, CA, USA) as an indicator of the amounts of protein loaded. The membranes were incubated for $1 \mathrm{~h}$ with a 1:1,000 dilution of horseradish peroxidase-conjugated donkey anti-rabbit immunoglobulin G (sc-45106; Santa Cruz Biotechnology, Inc.). The membranes were developed with a horseradish peroxidase chemiluminescence detection reagent (ECL Plus System), and then exposed to Hyperfilm ECL (both from Amersham Biosciences).

RNA extraction, reverse transcription and real-time PCR. Total RNA was extracted using TRIzol solution (Invitrogen, China) according to the manufacturer's instructions. Reverse transcription was performed in a $25 \mu \mathrm{l}$ reaction system with $2 \mu \mathrm{g}$ of total RNA-treated with M-MLV reverse transcriptase to synthesize first-strand cDNA (Promega, Madison, WI, USA) according to the manufacturer's recommendation. The cDNA was quantified by quantitative polymerase chain reaction (q-PCR). The primer sequences used in our study were: ZFN703 (F, 5'-GATCAGGGTCCTGAAGATGC-3 and R, 5'-CCGAGTTGAGTTTGGAGGAG-3'); $\beta$-actin (F, 5'-GCAT CCCCCAAAGTTCACAA-3' and R, 5'-AGGACTGGGCCA TTCTCCTT-3'). q-PCR was performed in a 96-well plate using LightCycler ${ }^{\circledR} 480$ real-time PCR system (Roche Diagnostics, Germany). Reaction volume was $20 \mu \mathrm{l}$ and contained $1 \mathrm{X}$ LightCycler ${ }^{\circledR} 480$ high-resolution melting master mix, $2.5 \mathrm{mM}$ of $\mathrm{MgCl}_{2}$, primers and cDNA. It was run under the following program: $95^{\circ} \mathrm{C}$ for $2 \mathrm{~min}, 43$ cycles of $95^{\circ} \mathrm{C}$ for $10 \mathrm{sec}, 58^{\circ} \mathrm{C}$ for $10 \mathrm{sec}, 72^{\circ} \mathrm{C}$ for $10 \mathrm{sec}$, then $72^{\circ} \mathrm{C}$ for $5 \mathrm{~min}$. The level of the test gene was compared with the housekeeping gene $\beta$-actin. The results are presented as relative gene expression compared with $\beta$-actin using $2^{-\Delta \Delta \mathrm{Ct}}$. The products of $\mathrm{q}-\mathrm{PCR}$ were verified on agarose gels.

Small interfering RNA (siRNA)-mediated gene silencing of ZNF703. The siRNAs to human ZNF703 were chemically synthesized by Shanghai GenePharma Co., Ltd. The siRNA sequences for the indicated genes were designed as follows: F, 5'-GCUUGACCCUGCCGGGAUU-3' and R, 5'-CUU ACAAGCUGGGAAUAUA-3'. The negative control dsRNA were as follows: F, 5'-UUCUCCGAACGUGUCACGUTT-3' and R, 5'-ACGUGACACGUUCGGAGAATT-3'. According to the manufacturer's specifications, the transfection of siRNA-ZNF703 was achieved with Lipo 2000 (Invitrogen, Gaithersburg, MD, USA) in 6-well plates. Exponential growth phase cells were plated in 6-well plates at a density of $2 \times 10^{5}$ cells $/ \mathrm{ml}$, cultured for $48 \mathrm{~h}$ and transfected with $1 \mu \mathrm{g}$ of siRNA in reduced serum medium (OPTI-MEM-I; Invitrogen) according to the manufacturer's protocol at 30-50\% confluence.

In vitro cell growth assay. The cells were prepared at a concentration of $1 \times 10^{4}$ cells $/ \mathrm{ml}$. Aliquots $(100 \mu \mathrm{l})$ were dispensed into 96-well plates. The cells were incubated for 1-7 days, and the 3-(4,5-dimethylthiazol-2-yl)-2,5-diphenyltetrazolium bromide (MTT) assay was performed by adding $20 \mathrm{ml}$ of MTT $(5 \mathrm{mg} / \mathrm{ml}$; Promega) for $4 \mathrm{~h}$. The supernatants were then removed. A total of $150 \mu \mathrm{l}$ of dimethylsulfoxide (Sigma, St. Louis, MO, USA) was added to each well. Fifteen minutes later, the absorbance value [optical density (OD)] of each well 
Table I. ZNF703 expression in gastric adenocarcinoma tissues and normal gastric mucosa.

\begin{tabular}{|c|c|c|c|c|c|}
\hline & \multirow[b]{2}{*}{$\mathrm{n}$} & \multicolumn{3}{|c|}{ ZNF703 } & \multirow[b]{2}{*}{ P-value } \\
\hline & & $-(\%)$ & $+(\%)$ & $++(\%)$ & \\
\hline Normal gastric mucosa & 25 & $22(88.0)$ & $3(12.0)$ & $0(0)$ & $<0.01$ \\
\hline Total carcinoma & 120 & $16(13.3)$ & $51(42.5)$ & $53(44.2)$ & \\
\hline Differentiation & & & & & 0.489 \\
\hline Intestinal & 67 & $6(9)$ & $31(46.3)$ & $30(44.8)$ & \\
\hline Diffuse & 53 & $10(18.9)$ & $20(37.7)$ & $23(43.4)$ & \\
\hline Depth of invasion & & & & & $<0.01$ \\
\hline T0 & 15 & $12(80)$ & $2(13.3)$ & $1(6.7)$ & \\
\hline $\mathrm{T} 1$ & 9 & $3(33.3)$ & $4(44.4)$ & $2(22.2)$ & \\
\hline $\mathrm{T} 2$ & 28 & $1(3.6)$ & $17(60.7)$ & $10(35.7)$ & \\
\hline T3 & 37 & $0(0)$ & $15(40.5)$ & $22(59.5)$ & \\
\hline $\mathrm{T} 4$ & 31 & $0(0)$ & $13(41.9)$ & $18(58.1)$ & \\
\hline Node metastasis & & & & & $<0.01$ \\
\hline N0 & 17 & $11(64.7)$ & $5(29.4)$ & $1(5.9)$ & \\
\hline N1 & 46 & $4(8.7)$ & $26(56.5)$ & $16(34.8)$ & \\
\hline $\mathrm{N} 2$ & 32 & $1(3.1)$ & $11(34.4)$ & $20(62.5)$ & \\
\hline N3 & 25 & $0(0)$ & $9(36)$ & $16(64)$ & \\
\hline Venous invasion & & & & & $<0.01$ \\
\hline Present & 73 & $15(20.5)$ & $36(49.3)$ & $22(30.1)$ & \\
\hline Absent & 47 & $1(2.1)$ & 15 (31.9) & $31(66)$ & \\
\hline
\end{tabular}

was measured at $490 \mathrm{~nm}$ with a microplate reader. All experiments were repeated three times.

In vitro migration analysis. A $0.1 \mathrm{ml}$ aliquot of cell suspension at $1 \times 10^{5}$ cells $/ 100 \mu \mathrm{l}$ was seeded on the upper chamber of Transwell filters (8- $\mu \mathrm{m}$ pore) (Corning Star, Cambridge, MA, USA). FCS-containing medium as a chemoattractant was added to the lower chamber and incubated for $18 \mathrm{~h}$ at $37^{\circ} \mathrm{C}$. Non-migrating cells that remained on the upper surface of the filter were removed, and the migrated cells on the lower surface of the filter were fixed with $100 \%$ methanol and stained. Five different fields were counted with a light microscope (Nikon E400) at a magnification x200. All assays were performed in triplicate.

Statistical analysis. Statistical software SPSS13.0 was used for analysis. Analyses comparing the degrees of ZNF703 expression were performed by the Mann-Whitney $U$ and Kruskal-Wallis H tests.

\section{Results}

Overexpression of ZNF703 in human gastric adenocarcinoma tissues/immunohistochemistry, western blot analysis and RT-PCR. We have summarized the immunohistochemical results in Table I in the expressions in human gastric adenocarcinoma and normal gastric mucosa. The expressed ZNF703 was localized in the cytoplasm and karyon of the cancer cells (Fig. 1). ZNF703 was expressed in 12\% (3/25) of normal gastric mucosa. Compared with these normal tissues, a rela- tively high expression level of ZNF703 was observed in $86.7 \%$ $(104 / 120)$ of gastric adenocarcinoma $(\mathrm{p}<0.001)$. ZNF703 expression in 12 gastric adenocarcinoma tissues and paired normal gastric mucosa was detected by RT-PCR and western blotting for verification (Fig. 2). Despite inter-individual variations in the extent of ZNF703 expression, the mRNA and protein was found to be significantly upregulated in gastric adenocarcinoma tissues ( $\mathrm{p}=0.03$ and $\mathrm{p}<0.01$, respectively).

Increased expression of ZNF703 is correlated with gastric adenocarcinoma progression. In ZNF703 expression, the invasive parts of the gastric adenocarcinoma tumor were intensely stained, as contrasted with the superficial area of the tumor in cases of invasive carcinomas. The ZNF703 overexpression was closely associated with the depth of invasion, lymph node metastasis, venous invasion (Table I).

Expression of ZNF703 in gastric adenocarcinoma cell lines/ western blot analysis. The expression of ZNF703 protein by normal gastric cell and gastric carcinoma cell lines was examined by western blot analysis. All four gastric carcinoma cell lines, AGS, SGC7901, MGC823 and BGC802, expressed ZNF703 protein. SGC7901 and BGC802 cells highly expressed ZNF703, while AGS and MGC823 cells expressed ZNF703 at low levels. However, the normal gastric cell line, GES, did not express ZNF703 protein. (Fig. 3).

In vitro silencing of ZNF703 inhibits the proliferation of gastric adenocarcinoma cells. We investigated the role of ZNF703 in the aggressive phenotypes of SGC7901 cells 

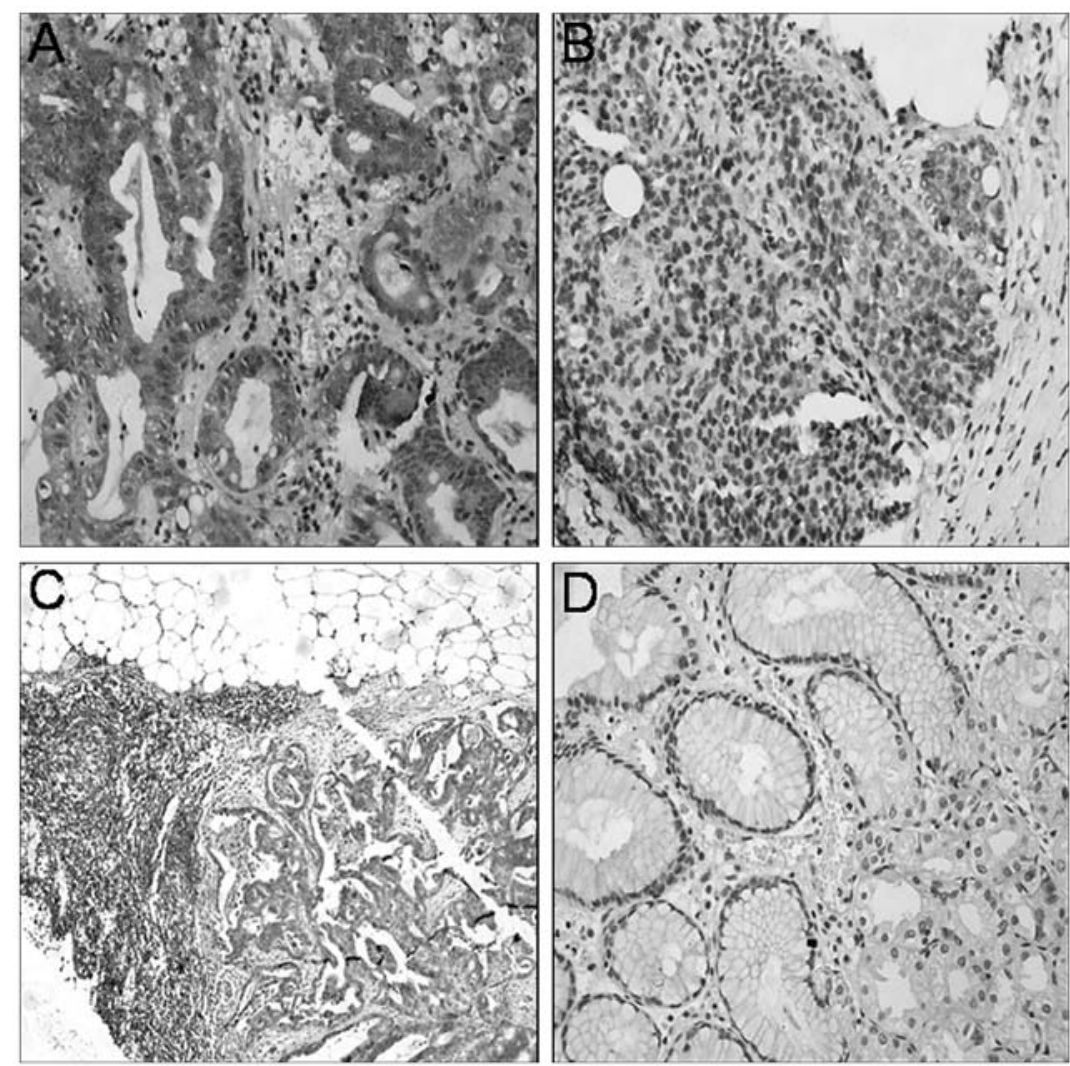

Figure 1. Immunohistochemical staining in human gastric carcinoma and normal gastric mucosa. (A, B and D, x200, DAB staining; C, x100, DAB staining) (A) Intestinal gastric carcinoma; (B) diffuse gastric carcinoma; (C) tumor-invaded lymph node; (D) normal gastric mucosa.
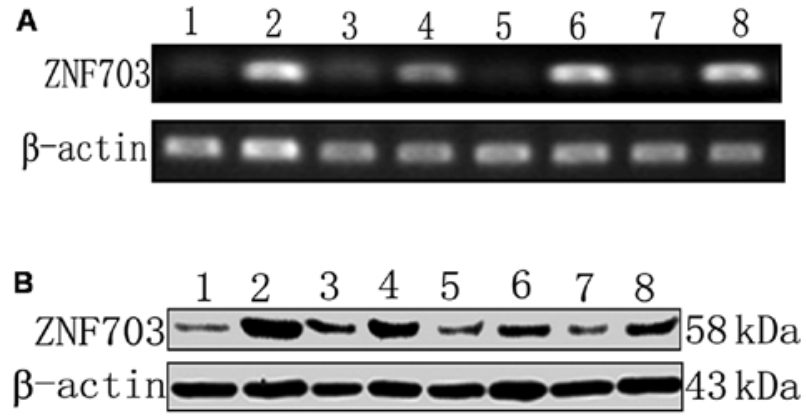

Figure 2. Investigation of ZNF703 in gastric adenocarcinoma tissues and normal gastric mucosa. (A) qRT-PCR and (B) western blotting analysis of ZNF703 in gastric adenocarcinoma tissues and paired normal gastric mucosa. Lane 1, case 1-normal gastric mucosa; lane 2, case 1-adenoma lane 3, case 2-normal gastric mucosa; lane 4, case 2-adenoma; lane 5, case 3-normal gastric mucosa; lane 6, case 3-adenoma; lane 7, case 4-normal gastric mucosa and lane 8 , case 4-adenoma.

in vitro. siRNA transfection was employed to knock down ZNF703 expression in SGC7901 cells, which had high endogenous ZNF703 expression. The effect of siRNA transfection on the expression of ZNF703 was confirmed by RT-PCR and western blot analysis (Fig. 4). The amount of ZNF703-1 mRNA and protein, normalized by $\beta$-actin, was reduced up to 80 and $70 \%$ respectively, compared with the negative control cells. A significantly slower proliferation was observed in ZNF703 siRNA-transfected cells when compared with control cells (Fig. 5).

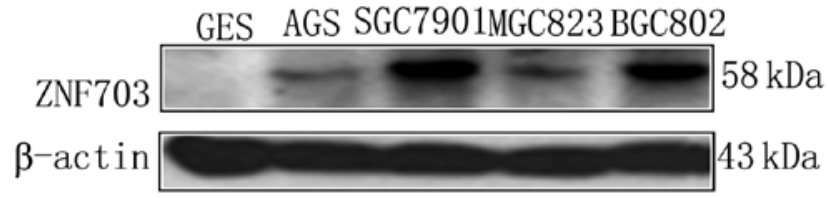

Figure 3. Expression of ZNF703 in human gastric carcinoma cell lines and normal gastric cell line.

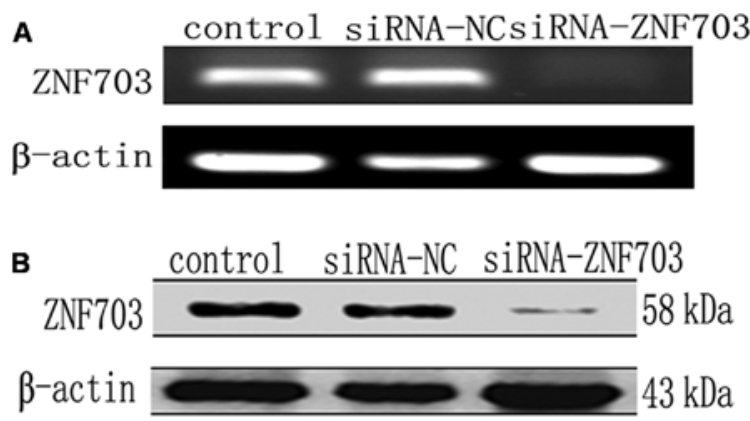

Figure 4. ZNF703 is significantly inhibited by siRNA-ZNF703 but not by the negative control siRNA in SGC7901 cells verified by (A) qRT-PCR and (B) western blotting.

In vitro silencing of ZNF703 inhibits the migration of gastric adenocarcinoma cells. To assess whether ZNF703 participates in gastric carcinoma migration, we performed Transwell assays on the SGC7901 cells transfected with the inhibitory 


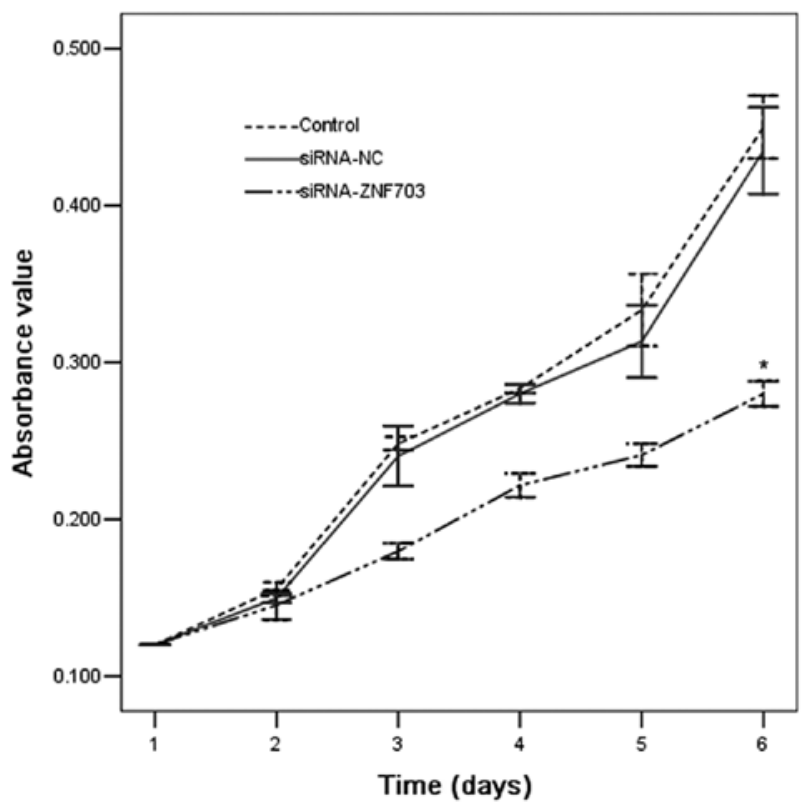

Figure 5. In vitro silencing of ZNF703 inhibits proliferation in SGC7901 cells.

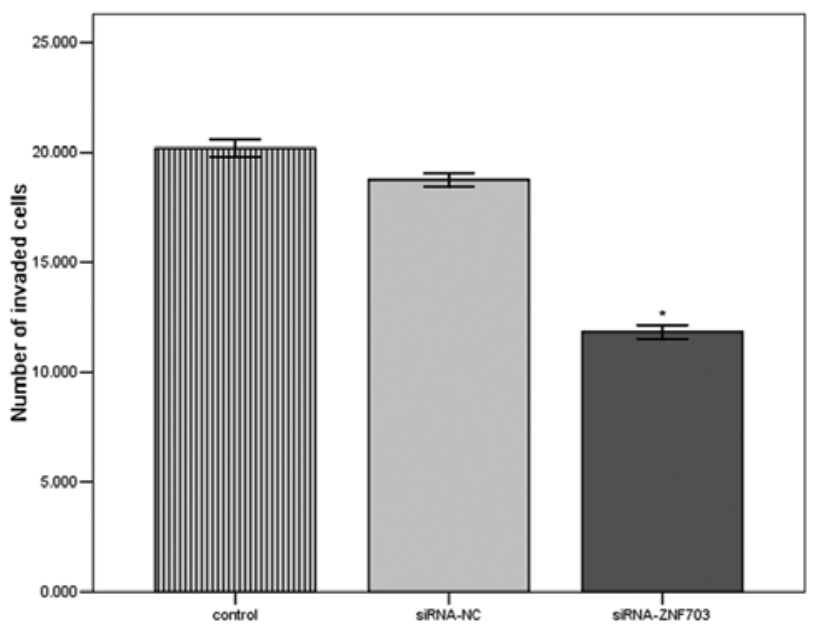

Figure 6. In vitro silencing of ZNF703 inhibits migration in SGC7901 cells.

siRNA-ZNF703 or the corresponding NC siRNA. Following transfection, siRNA-ZNF703 strongly reduced cell migration compared to the siRNA-NC and untreated controls, suggesting that ZNF703 was necessary for cell migration. Similar results were obtained in 3 independent experiments (Fig. 6).

\section{Discussion}

The occurrence and progression of cancer is considered to be a series of genetic events affecting the structure and/or expression of a number of oncogenes, anti-oncogenes and growth factors (1). In the present study, we demonstrated for the first time the expression of the ZNF703 protein in a series of 120 gastric adenocarcinoma tissues. The results showed that the expression level of ZNF703 protein was higher in gastric adenocarcinoma tissues than in normal gastric mucosa indicated by immunoblotting and immunohistochemistry study. ZNF703 may play a role in the invasive property of gastric carcinomas as there was a significant correlation between ZNF703 expression and depth of tumor invasion, lymph node metastasis. In the cases where the presence of venous invasion was noted, ZNF703 was more highly expressed. Histologically, the diffuse type of adenocarcinomas, which showed invasive spread, had a significantly poorer prognosis than the intestinal type (18). However, our study did not observe a significant correlation between ZNF703 expression and histological differentiation of human gastric carcinoma. It appears that gastric carcinoma and its demonstrated invasive properties are consistent with what has been conceptualized in multistep, a generally accepted theory with respect to cancer metastasis. Oncogenous biological evidence of ZNF703 was obtained in a loss-of-function experiment. Silencing the highly expressed ZNF703 by RNAi in gastric carcinoma SGC7901 cells led to reduced cell proliferation and migration. The results reported here suggest that ZNF703 has a role in invasion of gastric carcinoma, and constitutes one of the steps in the development of invasive characteristics.

Consistent with the high expression of ZNF703 in gastric cancer, previous data demonstrated a compelling case for amplification of ZNF703 as an important contributor to aggressive tumor behavior in a subset of estrogen receptor-positive breast cancers. Sircoulomb et al (11) showed that amplification of the ZNF703 gene, located in chromosomal region 8p12, preferentially occurred in luminal B tumors. Using mass spectrometry, they identified ZNF703 as a co-factor of a nuclear complex comprising DCAF7, PHB2 and NCOR2. ZNF703 expression resulted in the activation of stem cell-related gene expression leading to an increase in cancer stem cells. Holland et al (10) found ZNF703 amplification was associated with higher grade and more aggressive tumors (luminal B breast tumors), explaining the poorer clinical prognosis. Recently, Reynisdottir et al found that high ZNF703 expression, independent of the amplification, correlated with poorer prognosis for the breast cancer patients with ER-positive luminal tumors, particularly of the luminal B subtype (19). Furthermore, ectopic ZNF703 expression may transform NIH 3T3 mouse fibroblasts and regulate the proliferation in breast epithelial cells. Slorach et al showed that overexpression of the mouse ortholog of ZNF703, Zeppo1 (Zpol), in an allograft model using the metastatic mouse mammary tumor cell line 4T1 promoted tumor invasion and metastasis, further implicating ZNF703 as a likely contributor to cancer pathogenesis (13). However, the potential mechanism of ZNF703 in carcinogenesis was unclear and little research has been conducted to explore this molecular mechanism (20). ZNF703 may act as a transcriptional repressor $(21,22)$ and affect target genes which mediate various aspects of cancer pathophysiology, such as EMT, adhesion, motility, differentiation (23), proliferation (24) and cancer stem cell (CSC) renewal, CDH1, cadherin-1, CTTND1, p120-catenin and TGFBR2 $(10,13,25)$.

In conclusion, we observed that ZNF703 was upregulated in gastric adenocarcinoma, especially in cases with invasive properties, indicating its relationship with poor clinical outcome. Silencing the highly expressed ZNF703 by RNAi inhibited cancer cell growth and migration in vitro. Although the molecular mechanism of ZNF703 in carcinogenesis remains to be explored, it should be considered a therapeutic target for metastatic gastric cancer. 


\section{Acknowledgements}

This study was supported by grants from Guangdong Provincial Science and Technology Projects (nos. 2010B031600243, 2011B05040009 and 2012B050600020), and the National Natural Science Foundation of China (no. 81272761).

\section{References}

1. Hartgrink HH, Jansen EP, van Grieken NC and van de Velde CJ: Gastric cancer. Lancet 374: 477-490, 2009.

2. Wagner AD, Grothe W, Haerting J, Kleber G, Grothey A and Fleig WE: Chemotherapy in advanced gastric cancer: a systematic review and meta-analysis based on aggregate data. J Clin Oncol 24: 2903-2909, 2006.

3. Goseki N, Koike M and Yoshida M: Histopathologic characteristics of early stage esophageal carcinoma. A comparative study with gastric carcinoma. Cancer 69: 1088-1093, 1992.

4. Nakamura M, Runko AP and Sagerström CG: A novel subfamily of zinc finger genes involved in embryonic development. J Cell Biochem 93: 887-895, 2004.

5. Dorfman R, Glazer L, Weihe U, Wernet MF and Shilo BZ: Elbow and Noc define a family of zinc finger proteins controlling morphogenesis of specific tracheal branches. Development 129: 3585-3596, 2002.

6. Zhao X, Yang Y, Fitch DH and Herman MA: TLP-1 is an asymmetric cell fate determinant that responds to Wnt signals and controls male tail tip morphogenesis in C. elegans. Development 129: 1497-1508, 2002.

7. Runko AP and Sagerström CG: Nlz belongs to a family of zinc-finger-containing repressors and controls segmental gene expression in the zebrafish hindbrain. Dev Biol 262: 254-267, 2003.

8. Gerhard DS, Wagner L, FeingoldEA, et al: The status, quality, and expansion of the NIH full-length cDNA project: the Mammalian Gene Collection (MGC). Genome Res 14: 2121-2127, 2004.

9. Chin K, DeVries S, Fridlyand J, et al: Genomic and transcriptional aberrations linked to breast cancer pathophysiologies. Cancer Cell 10: 529-541, 2006.

10. Holland DG, Burleigh A, Git A, et al: ZNF703 is a common Luminal B breast cancer oncogene that differentially regulates luminal and basal progenitors in human mammary epithelium. EMBO Mol Med 3: 167-180, 2011.

11. Sircoulomb F, Nicolas N, Ferrari A, et al: ZNF703 gene amplification at 8p12 specifies luminal B breast cancer. EMBO Mol Med 3: 153-166, 2011.
12. Eriksson N, Benton GM, Do CB, et al: Genetic variants associated with breast size also influence breast cancer risk. BMC Med Genet 13: 53, 2012.

13. Slorach EM, Chou J and Werb Z: Zeppol is a novel metastasis promoter that represses $E$-cadherin expression and regulates p120-catenin isoform expression and localization. Genes Dev 25: 471-484, 2011.

14. Lauren P: The two histological main types of gastric carcinoma: diffuse and so-called intestinal-type carcinoma. An attempt at a histo-clinical classification. Acta Pathol Microbiol Scand 64: 31-49, 1965.

15. Goseki N, Takizawa T and Koike M: Differences in the mode of the extension of gastric cancer classified by histological type: new histological classification of gastric carcinoma. Gut 33: 606-612, 1992

16. Fenoglio-Preiser CM, Noffsinger AE, Stemmermann GN, et al: Gastrointestinal Pathology: An Atlas and Text. Raven Press, New York, 1989.

17. Hamilton SR and Aaltonen LA (eds): Pathology and Genetics Tumours of the Digestive System. World Health Organization Classification of Tumours. IARC Press, Lyon, 2000.

18. Trainer DL, Kline T, McCabe FL, et al: Biological characterization and oncogene expression in human colorectal carcinoma cell lines. Int J Cancer 41: 287-296, 1988.

19. Reynisdottir I, Arason A, Einarsdottir BO, et al: High expression of ZNF703 independent of amplification indicates worse prognosis in patients with luminal B breast cancer. Cancer Med 2: 437-446, 2013

20. Spellman $P$ and Gray J: A new treasure in the breast cancer gene hunt. Nat Med 17: 422-423, 2011.

21. Nakamura M, Choe SK, Runko AP, Gardner PD and Sagerström CG: Nlz1/Znf703 acts as a repressor of transcription. BMC Dev Biol 8: 108, 2008.

22. Pereira-Castro I, Costa AM, Oliveira MJ, et al: Characterization of human NLZ1/ZNF703 identifies conserved domains essential for proper subcellular localization and transcriptional repression. J Cell Biochem 114: 120-133, 2013.

23. Bazarov AV and Yaswen P: Who is in the driver's seat in $8 \mathrm{p} 12$ amplifications? ZNF703 in luminal B breast tumors. Breast Cancer Res 13: 308, 2011.

24. Zhang X, Mu X, Huang O, et al: Luminal breast cancer cell lines overexpressing ZNF703 are resistant to tamoxifen through activation of Akt/mTOR signaling. PLoS One 8: e72053, 2013.

25. Gelsi-Boyer V, Orsetti B, Cervera N, et al: Comprehensive profiling of $8 \mathrm{p} 11-12$ amplification in breast cancer. Mol Cancer Res 3: 655-667, 2005. 